\title{
Pediatric Nurses' and Parents' Attitudes Regarding the Importance of Families' Involvement in Care given for their Children
}

Amina Mohamed Thabet ${ }^{1} \&$ Nora Abd-Elhamid Zaki ${ }^{2}$.

1. Assistant professor of Pediatric Nursing, Faculty of Nursing, Sohag University, Egypt.

2. Assistant professor of Pediatric Nursing, Faculty of Nursing, Assiut University, Egypt.

\begin{abstract}
Background: Contribution of families in child care is considered necessary for meeting children's needs and for the well-being of the whole family. This study aimed to assess pediatric nurses' and parents' attitudes regarding the importance of families' involvement in care given for their children. Descriptive correlation research design was utilized in this study. Convenience sampling composed of 100 pediatric nurses and 100 parents accompanied their children at inpatient pediatric departments from January to February 2017. Two tools were used to collect data as mothers' attitude scale and families' importance in nursing care-nurses' attitudes scale. Results: Pediatric nurses and parents' showed neutral attitude $(77 \%$ \& $43 \%$ respectively) and the rest of them were between negative and positive attitude. Highly statistical significant difference were found between pediatric nurses and parent's attitudes $\left(\mathrm{p}<0.001^{* *}\right)$ concerning the importance of families' involvement in nursing care Conclusion: The present study concluded that, more than three fourths of pediatric nurses and nearly half of parents showed neutral attitude and highly statistical significance differences were found between pediatric nurses' and parents' socio demographic and their attitude regarding the importance of families involvement in care given for their children. Most of parents reported that they prefer the presence beside their children in any of the nursing procedures otherwise painful procedures. Recommendation: In service training programs should be provided for all categories of pediatric nurses regarding the importance of involving families in nursing care.
\end{abstract}

Key words: Pediatric nurses, Parents, Attitudes, Family involvement, Care given \& Children.

\section{Introduction}

Hospitalization can be an intimidating and stressful experience for children because they are unaccustomed with the environment and medical procedures and ignorant of the reasons for hospitalization. It can result in children's anger, vagueness, anxiety and feelings of helplessness. Anxiety hinders children's efficacy in coping with medical treatment and increases their uncooperative behavior and negative feelings towards healthcare professionals (Chung et al., 2016) Mothers of the hospitalized children hoped to be respected by the pediatric nurses, to participate in the nursing of their children, and to receive enough information to work together with pediatric nurses, but felt that they were not respected by the pediatric nurses or did not obtain enough information (Kim \& Cho, 2017).

The child profits greatly from mother or caregiver existence and participation and experiences less emotional distress and anxiety. If the mother-child attachment remnants uninterrupted, the child experiences less behavioral maladjustment as a result of hospitalization. Mothers often need to support their child before, during and immediately after medical or nursing procedures and their presence proposals reassurance and easiness to the child. During invasive procedures, mothers can be educated to support the child by provided that gentle touch, talking, singing, giving reassurance, or demonstrating stress decrease techniques (Ball, et al., 2012).

The family generally has a wish to be a part of the health care, also a need of good communication and a close cooperation with pediatric nurses to gain a better understanding about their family member's state (Holmgren, et al., 2014) It is necessary that health care professionals should unite parents into their strategic practices in order to strengthen the involvement and participation of parents in care delivery, according to the needs of each child and family, sharing knowledge, promoting healthiness, and contributing to humanized and quality healthcare (Pinheiro de Melo et al., 2014).

Pediatric nurses in approximately all health care environment meet families in their daily work. The quality of these meetings is influenced by pediatric nurses' attitudes about the importance of comprising families in nursing care (Benzein et al., 2008). The attendance of families in the health care environment is an essential stimulus to activates pediatric nurses' attitude towards them, and their attitudes are based on feelings and emotions (affective element), thoughts and beliefs (cognitive component), and reaction tendencies (behavioral component) (Oliveira et al., 2011) Pediatric nurses would not see the presence of the hospitalized child's family as a load, but rather have a flexible attitude so that they can reverence 
them as spouses in cooperation (Saveman et al., 2011).

One indicator used to assess the quality of the relationship between health professionals and family members is to assess the attitudes towards the participation of families in care (Bell and Wright, 2011).

\section{Significance of the study}

It is nowadays well-accepted that pediatric care should be provided within the context of families, with parents who are considered necessary partners in their children's care. Pediatric health care has shifted to family centered-care that is based on close and incessant involvement of the child's family members (Mohammed et al., 2013). Family-centered care practices proliferation retrieval the sense of control lost in child care, reinforcement communication between parents and health care personnel (Dur et al., 2016), positively affect mother-child mental health and rise parents' satisfaction (Atay et al., 2011).

\section{Aim of the study}

The aim of this study was to assess pediatric nurses' and parents' attitudes regarding the importance of families' involvement in care given for their children

\section{Research questions}

1- What are the pediatric nurses' and parents' attitudes regarding importance of families' involvement in care given for their children?

2- What are the relation between parents' attitudes and pediatric nurses' attitudes regarding importance of families' involvement in care given for their children?

\section{Subjects \& Method \\ Research design}

A descriptive correlation research design was used to fit the aim of this study.

\section{Setting}

The study was conducted at four pediatric nursing departments (medical, surgical, rehydration, and emergency) in Assuit University Children Hospital.

\section{Sample}

A convenience sample composed of 100 pediatric nurses (33 medical, 26 surgical, 17 rehydration, and 24 emergency) and 100 parents (25 parents in each department) who are attending the pediatric departments with their children through the data collection period, all of them were called to participate in the study.

\section{Data Collection Tools}

Two tools were used for collecting the essential data as the following: Tool I: Mothers' attitude scale: It was developed by
Mohammed et al., (2013) to collect the needed data which translated into the Arabic form by the researchers. It consisted of two parts:

Part I: Socio-demographic Characteristics of Parents, such as age, level, of education, marital status, number of children and residence. It involved data about children as, child's age, gender, birth order, diagnosis, and history of pervious hospitalization.

Part II: This part composed of 18 questions related mothers' attitudes used to assess the mothers' attitudes regarding importance of families' involvement in care given for their children.

Scoring system of tool I: consisted of three point's likert scale items: agree, neutral and disagree.

Each statement was scored as agree answers (2), neutral answers (1) and answers disagree (0). The total attitude was calculated as follows. The mean of total score for all items of agreement answer was considered as a positive attitude (more than 70\%), while, disagree considered as a negative attitude (less than $60 \%$ ), and neutral was considered as neutral attitude (from $60 \%$ to $70 \%$ ).

Tool II: Families' Importance in Nursing CarePediatric nurses' Attitudes (FINC-NA). This tool has been developed and psychometrically tested by Benzein et al., (2008) which translated into the Arabic form by the researchers. It consisted of two parts:

Part I: Socio-demographic Characteristics of the Pediatric Nurses such as: age, qualification, job title, marital status, years of experiences and training courses related to family involvement in care and parent participation in the care of hospitalized children.

Part II: Families' Importance in Nursing CarePediatric Nurses' Attitudes (FINC-NA) tool to measures pediatric nurses' attitudes about the importance of including families in nursing care. The FINC-NA consists of 26 items, and the responses alternatives are constructed as a four-point Likert scale (strongly agree, agree, disagree, and strongly disagree).

\section{Scoring system of tool II}

The score ranges from 1 to 4 for each item and ranges from 26 to 104 for the whole tool. Strongly agree (4), agree (3), disagree (2), and strongly disagree (1).

The scale classified into the following subscales: Family as a resource in nursing care (Fam-RNC) (containing10 items with a possible score range of 140); family as a conversational partner (Fam-CP) (encompassing 8 items with a possible score range of 1-32); family as a burden (Fam-B) (comprising 4 items with a possible score range of 1-16); and lastly family as its own resource (Fam-OR) (including 4 items with a possible range of 1-16). 
The levels of parents and pediatric nurses general attitudes were divided into three categories as negative attitude (less than 60\%), neutral attitude (from $60 \%$ to $70 \%$ ) and positive attitude (more than $70 \%$ ).

Pilot Study: was carried out on $10 \%$ of participants (10 mothers) and (10 nurses), to test the transparency and feasibility of questions and for time estimation required for the interview and added to the total sample.

Validity and Reliability: As the two tools translated into Arabic form, the internal consistency estimated with crombach's alpha:

The reliability for tool I (Parents' attitude) was 0.690 and its validity was $83 \%$ (No .item 18 ).

The reliability for tool II (Pediatric nurses' attitude) was 0.702 and its validity was $0.84 \%$ (No of items 26).

\section{Data Collection Procedure}

1. An official letter to carry out the study was directed from the Faculty of Nursing, Assuit University to the hospital administrative authorities in order to obtain their acceptance to collect necessary data from the previous settings. Then, the permission was obtained from the hospital administrative authority.

2. Data were collected in a period of two months (from the beginning of January to the end of February 2017).

3. In order to increase the compliance, explanation about the nature of the study, its aim and benefits.

4. Written consent was achieved from all parents and pediatric nurses from the previous departments to participate in the study.

5. After receiving permission from the parents and pediatric nurses, the designed questionnaire sheets was distributed to one hundred parents and one hundred pediatric nurses to assess their attitudes concerning the importance of family presence in pediatric nursing care.

6. Collecting the data from the pediatric nurses was conducted individually at the morning and afternoon shifts to prevent work interruption.

7. Each pediatric nurse was asked to fill the data collection tools individually. The time needed for each pediatric nurse ranged from 20-25 minutes.

8. Collecting the data from the parents was conducted, at the same time when the researchers present at study setting. Each parent was interviewed individually. The time needed for each parent to answer the sheet ranged from 3035 minutes.

\section{Ethical considerations}

All parents and pediatric nurses were knowledgeable about the aim of study in order to attain their acceptance to participate in the current study. The researchers explained to them benefits of the current study. The researchers informed the parents and pediatric nurses that all data collected during the study were considered confidential. The researchers also informed the participants about their rights to withdraw from the study at any time without giving any reason.

\section{Statistical Analysis}

The data were tested for normality using the Anderson-Darling test and for homogeneity variances prior to further statistical analysis. Categorical variables were described by number and percent $(\mathrm{N}$, $\%$ ), where continuous variables described by mean and standard deviation (Mean, SD). Chi-square test and fisher exact test used to compare between categorical variables where compare. A two-tailed $\mathrm{p}$ $<0.05$ was considered statistically significant. All analyses were performed with the IBM SPSS 20.0 software. 


\section{Results}

Table (1): Percentage distribution of pediatric nurses related to socio- demographic data $(n=100)$.

\begin{tabular}{|l|c|c|}
\hline \multicolumn{1}{|c|}{ Item } & No. & \% \\
\hline Age in years: & & $\mathbf{8 1 . 0}$ \\
\hline $20<30$ & 81 & 9.0 \\
\hline $30<40$ & 9 & 10.0 \\
\hline$\geq 40$ Years & 10 & \\
\hline Sex & & $\mathbf{7 5 . 0}$ \\
\hline Female & 75 & 25.0 \\
\hline Male & 25 & \\
\hline Level of education: & & 33.0 \\
\hline Bachelor of nursing & 33 & $\mathbf{3 4 . 0}$ \\
\hline Secondary school diploma & 34 & 33.0 \\
\hline Diploma of technical institute & 33 & \\
\hline Clinical position: & & 26.0 \\
\hline Head pediatric nurse & 26 & $\mathbf{7 0 . 0}$ \\
\hline Supervisor & 70 & 4.0 \\
\hline Bedside pediatric nurse & 4 & \\
\hline Years of experience: & & $\mathbf{5 2 . 0}$ \\
\hline$<5$ years & 52 & 19.0 \\
\hline $5<10$ years & 19 & 29.0 \\
\hline$\geq 10$ Year or more & 29 & \\
\hline Training course of about family involvement in care: & & $\mathbf{6 5 . 0}$ \\
\hline Yes & 65 & 35.0 \\
\hline No & 35 & \\
\hline
\end{tabular}

Table 2: Percentage distribution of parents' and their children related to socio-demographic characteristic $(\mathbf{n}=100)$

\begin{tabular}{|l|c|c|}
\hline \multicolumn{1}{|c|}{ Item } & No. & \% \\
\hline Age (mothers and fathers): & & \\
\hline$<30$ years & 65 & 65.0 \\
\hline$\geq 30$ years & 35 & 35.0 \\
\hline Sex & 3 & \\
\hline Fathers & 97 & 97.0 \\
\hline Mothers & & \\
\hline Level of education & 73 & 73.0 \\
\hline Secondary & 16 & 16.0 \\
\hline Technical qualification & 11 & 11.0 \\
\hline University degree & & \\
\hline Residence & 32 & 32.0 \\
\hline Urban & 68 & 68.0 \\
\hline Rural & & \\
\hline Child been in hospital before & 52 & 52.0 \\
\hline Yes & 48 & 48.0 \\
\hline No & & \\
\hline Previous experience of hospitalization & 31 & 31.0 \\
\hline Yes & 69 & 69.0 \\
\hline No & & \\
\hline child's age & 89 & 89.0 \\
\hline $1<6$ years & 6 & 6.0 \\
\hline $6<12$ years & & \\
\hline
\end{tabular}




\begin{tabular}{|l|c|c|}
\hline \multicolumn{1}{|c|}{ Item } & No. & \% \\
\hline 12 to 18 years & 5 & 5.0 \\
\hline Gender of the child & & \\
\hline Male & 67 & 67.0 \\
\hline Female & 33 & 33.0 \\
\hline Child's birth order & & \\
\hline The first & 30 & 30.0 \\
\hline Second & 24 & 24.0 \\
\hline Third & 25 & 25.0 \\
\hline Fourth & 16 & 16.0 \\
\hline Fifth or more & 5 & 5.0 \\
\hline
\end{tabular}

Table 3: Percentage distribution of pediatric nurses' attitude regarding the importance presence of family in nursing care $(\mathbf{n}=\mathbf{1 0 0})$

\begin{tabular}{|l|c|c|c|c|c|c|c|c|}
\hline \multicolumn{1}{|c|}{ Items } & \multicolumn{2}{|c|}{$\begin{array}{c}\text { Strongly } \\
\text { agree }\end{array}$} & \multicolumn{2}{|c|}{ Agree } & \multicolumn{2}{c|}{ Disagree } & \multicolumn{2}{c|}{$\begin{array}{c}\text { Strongly } \\
\text { disagree }\end{array}$} \\
\hline \multicolumn{1}{|c|}{ I- Family as a resource in nursing care (Fam- } & No. & $\%$ & No. & $\%$ & No. & $\%$ & No. & $\%$ \\
\hline The presence of family members eases my workload & 43 & 43.0 & 32 & 32.0 & 21 & 21.0 & 4 & 4.0 \\
\hline $\begin{array}{l}\text { The presence of family members gives me a feeling } \\
\text { of security }\end{array}$ & 33 & 33.0 & 33 & 33.0 & 30 & 30.0 & 4 & 4.0 \\
\hline $\begin{array}{l}\text { The presence of family members is important to me } \\
\text { as a pediatric nurse }\end{array}$ & 18 & 18.0 & 56 & 56.0 & 22 & 22.0 & 4 & 4.0 \\
\hline $\begin{array}{l}\text { Family members should be invited to actively take } \\
\text { part in the patient's nursing care }\end{array}$ & 22 & 22.0 & 27 & 27.0 & 42 & 42.0 & 9 & 9.0 \\
\hline $\begin{array}{l}\text { Family members should be invited to actively take } \\
\text { part in planning patient care }\end{array}$ & 17 & 17.0 & 46 & 46.0 & 23 & 23.0 & 14 & 14.0 \\
\hline $\begin{array}{l}\text { A good relationship with family members gives me } \\
\text { job satisfaction }\end{array}$ & 24 & 24.0 & 44 & 44.0 & 23 & 23.0 & 9 & 9.0 \\
\hline $\begin{array}{l}\text { Getting involved with families gives me a feeling of } \\
\text { being useful }\end{array}$ & 0 & 0.0 & 31 & 31.0 & 65 & 65.0 & 4 & 4.0 \\
\hline $\begin{array}{l}\text { I gain a lot of worthwhile knowledge from families } \\
\text { which I can use in my work }\end{array}$ & 8 & 8.0 & 73 & 73.0 & 15 & 15.0 & 4 & 4.0 \\
\hline $\begin{array}{l}\text { The presence of family members is important for the } \\
\text { family members themselves }\end{array}$ & 32 & 32.0 & 40 & 40.0 & 28 & 28.0 & 0 & 0.0 \\
\hline It is important to spend time with families & 4 & 4.0 & 42 & 42.0 & 45 & 45.0 & 9 & 9.0 \\
\hline II- Family as a burden (Fam-B) & & & & & & & & \\
\hline $\begin{array}{l}\text { The presence of family members makes me feel that } \\
\text { they are checking up on me }\end{array}$ & 18 & 18.0 & 44 & 44.0 & 23 & 23.0 & 15 & 15.0 \\
\hline $\begin{array}{l}\text { The presence of family members makes me feel } \\
\text { stressed }\end{array}$ & 33 & 33.0 & 49 & 49.0 & 13 & 13.0 & 5 & 5.0 \\
\hline $\begin{array}{l}\text { The presence of family members holds me back in } \\
\text { my work }\end{array}$ & 29 & 29.0 & 29 & 29.0 & 37 & 37.0 & 5 & 5.0 \\
\hline I don't have time to take care of families & 49 & 49.0 & 28 & 28.0 & 23 & 23.0 & 0 & 0.0 \\
\hline
\end{tabular}


Table (3): Continue, percentage distribution of pediatric nurses' attitude regarding the importance presence of family in nursing care $(n=100)$.

\begin{tabular}{|c|c|c|c|c|c|c|c|c|}
\hline \multirow{2}{*}{$\begin{array}{l}\text { Items } \\
\text { III- Family as a conversational partner (Fam-CP) }\end{array}$} & \multicolumn{2}{|c|}{$\begin{array}{l}\text { Strongly } \\
\text { agree }\end{array}$} & \multicolumn{2}{|c|}{ Agree } & \multicolumn{2}{|c|}{ Disagree } & \multicolumn{2}{|c|}{$\begin{array}{l}\text { Strongly } \\
\text { disagree }\end{array}$} \\
\hline & No & $\%$ & No & $\%$ & No & $\%$ & No & $\%$ \\
\hline $\begin{array}{l}\text { I invite family members to have a conversation at the } \\
\text { end of the care period }\end{array}$ & 19 & 19.0 & 58 & 58.0 & 14 & 14.0 & 9 & 9.0 \\
\hline $\begin{array}{l}\text { I ask family members to take part in discussions from the } \\
\text { very first contact, }\end{array}$ & 29 & 29.0 & 52 & 52.0 & 19 & 19.0 & 0 & 0.0 \\
\hline when a patient comes into my care & 18 & 18.0 & 54 & 54.0 & 28 & 28.0 & 0 & 0.0 \\
\hline I always find out what family members a patient has & 27 & 27.0 & 50 & 50.0 & 15 & 15.0 & 8 & 8.0 \\
\hline $\begin{array}{l}\text { I invite family members to speak about changes in the } \\
\text { patient's condition }\end{array}$ & 23 & 23.0 & 50 & 50.0 & 14 & 14.0 & 13 & 13.0 \\
\hline I invite family members to speak when planning care & 32 & 32.0 & 46 & 46.0 & 22 & 22.0 & 0 & 0.0 \\
\hline $\begin{array}{l}\text { It is important to find out what family members a patient } \\
\text { has }\end{array}$ & 23 & 23.0 & 69 & 69.0 & 8 & 8.0 & 0 & 0.0 \\
\hline $\begin{array}{l}\text { I invite family members to actively take part in the } \\
\text { patient's care }\end{array}$ & 34 & 34.0 & 33 & 33.0 & 33 & 33.0 & 0 & 0.0 \\
\hline \multicolumn{9}{|l|}{ IV-Family as own resource (Fam-OR) } \\
\hline $\begin{array}{l}\text { I encourage families to use their own resources so that } \\
\text { they have the optimal possibilities to cope with situations } \\
\text { by themselves }\end{array}$ & 34 & 34.0 & 48 & 48.0 & 18 & 18.0 & 0 & 0.0 \\
\hline $\begin{array}{l}\text { I see myself as a resource for families so that they can } \\
\text { cope as well as possible with their situation }\end{array}$ & 28 & 28.0 & 49 & 49.0 & 23 & 23.0 & 0 & 0.0 \\
\hline I consider family members as co-operating partners & 14 & 14.0 & 78 & 78.0 & 8 & 8.0 & 0 & 0.0 \\
\hline I ask families how I can support them & 13 & 13.0 & 67 & 67.0 & 20 & 20.0 & 0 & 0.0 \\
\hline
\end{tabular}

Table (4): Mean \pm SD of the general pediatric nurses' attitude as regards FINC-NA scale and the mean of its subscale $(\mathbf{n}=\mathbf{1 0 0})$

\begin{tabular}{|l|c|c|c|c|}
\hline \multicolumn{1}{|c|}{ Items } & No. & Score & Range & Mean \pm SD \\
\hline General pediatric nurses' attitude & 26 & 78 & $29-64$ & $49.6 \pm 6.96$ \\
\hline $\begin{array}{l}\text { Subscale: Family as a resource in nursing care (Fam- } \\
\text { RNC) }\end{array}$ & 10 & 30 & $4-23$ & $17.65 \pm 4.43$ \\
\hline Subscale: Family as a burden (Fam-B) & 8 & 24 & $9-21$ & $15.92 \pm 3.4$ \\
\hline Subscale: Family as a conversational partner (Fam-CP) & 4 & 12 & $4-11$ & $7.83 \pm 2.02$ \\
\hline Subscale: Family as own resource (Fam-OR) & 4 & 12 & $5-10$ & $8.2 \pm 1.51$ \\
\hline
\end{tabular}

Table (5): Percentage distribution of parents' attitude regarding the importance of family in pediatric nursing care $(\mathbf{n}=100)$.

\begin{tabular}{|l|c|c|c|c|c|c|}
\hline \multicolumn{1}{|c|}{ Items } & \multicolumn{2}{c|}{ Agree } & \multicolumn{2}{c|}{ Disagree } & \multicolumn{2}{c|}{ Neutral } \\
\cline { 2 - 7 } & No. & $\mathbf{\%}$ & No. & $\mathbf{\%}$ & No. & $\%$ \\
\hline $\begin{array}{l}\text { I prefer presence next to my child in any of the nursing } \\
\text { procedures. }\end{array}$ & 95 & 95 & 2 & 2 & 3 & 3 \\
\hline I prefer presence next to my child during invasive procedures. & 58 & 58 & 37 & 37 & 5 & 5 \\
\hline $\begin{array}{l}\text { I think that there is no need for my presence during invasive } \\
\text { painful procedures. }\end{array}$ & 75 & 75 & 15 & 15 & 10 & 10 \\
\hline I cannot see my child in pain during nursing procedures. & 63 & 63 & 26 & 26 & 11 & 11 \\
\hline $\begin{array}{l}\text { My child cries more in my presence during the painful nursing } \\
\text { procedures. }\end{array}$ & 38 & 38 & 51 & 51 & 11 & 11 \\
\hline My child usually restless during my stay. & 24 & 24 & 70 & 70 & 6 & 6 \\
\hline $\begin{array}{l}\text { I'm confident that the pediatric nurse doing what I'm doing to } \\
\text { reassure my child. }\end{array}$ & 51 & 51 & 34 & 34 & 15 & 15 \\
\hline
\end{tabular}




\begin{tabular}{|l|l|l|l|l|l|l|}
\hline $\begin{array}{l}\text { The pediatric nurse cannot be replaces the mother during painful } \\
\text { procedures. }\end{array}$ & 61 & 61 & 24 & 24 & 15 & 15 \\
\hline $\begin{array}{l}\text { The pediatric nurse can performed some painful procedures and } \\
\text { the child in his mother's lap. }\end{array}$ & 53 & 53 & 22 & 22 & 25 & 25 \\
\hline $\begin{array}{l}\text { The pediatric nurse works to relieve pain by play during } \\
\text { procedures instead of a mother. }\end{array}$ & 46 & 46 & 23 & 23 & 31 & 31 \\
\hline $\begin{array}{l}\text { The pediatric nurse works to relieve pain by play during the } \\
\text { painful procedures only. }\end{array}$ & 50 & 50 & 27 & 27 & 23 & 23 \\
\hline $\begin{array}{l}\text { The pediatric nurse prepares the mothers to support their children } \\
\text { during all nursing procedures. }\end{array}$ & 71 & 71 & 17 & 17 & 12 & 12 \\
\hline $\begin{array}{l}\text { The pediatric nurse prepares the mothers to support their children } \\
\text { during nursing procedures only. }\end{array}$ & 36 & 36 & 44 & 44 & 20 & 20 \\
\hline $\begin{array}{l}\text { The hospital administration makes preparation for children and } \\
\text { mothers at the beginning of hospitalization. }\end{array}$ & 40 & 40 & 34 & 34 & 26 & 26 \\
\hline $\begin{array}{l}\text { Some pediatric nurses do not prefer the presence of the mother } \\
\text { during any of the nursing procedures. }\end{array}$ & 39 & 39 & 39 & 39 & 22 & 22 \\
\hline $\begin{array}{l}\text { All pediatric nurses do not prefer the presence of the mother } \\
\text { during any of the nursing procedures. }\end{array}$ & 20 & 20 & 63 & 63 & 17 & 17 \\
\hline $\begin{array}{l}\text { Some pediatric nurses do not prefer the presence of the mother } \\
\text { during invasive nursing procedures. }\end{array}$ & 32 & 32 & 50 & 50 & 18 & 18 \\
\hline $\begin{array}{l}\text { All pediatric nurses do not prefer the presence of the mother } \\
\text { during invasive nursing proceed }\end{array}$ & 25 & 25 & 45 & 45 & 30 & 30 \\
\hline
\end{tabular}

Table (6): Parents versus Pediatric nurses' general attitude regarding the importance of family presence in nursing care with children.

\begin{tabular}{|l|c|c|c|c|c|c|}
\hline \multirow{2}{*}{ General attitude } & \multicolumn{2}{|c|}{$\begin{array}{c}\text { Parents' attitude } \\
\text { (N=100) }\end{array}$} & \multicolumn{2}{|c|}{$\begin{array}{c}\text { Pediatric nurses' } \\
\text { attitude (N=100) }\end{array}$} & \multirow{2}{*}{$\mathrm{X}^{2}$} & \multirow{2}{*}{$\mathrm{P}$} \\
\cline { 2 - 5 } & No. & $\%$ & No. & $\%$ & & \\
\hline \hline Negative & 23 & 23.0 & 9 & 9.0 & 24.09 & $<0.001^{* *}$ \\
\hline Neutral & $\mathbf{4 3}$ & $\mathbf{4 3 . 0}$ & $\mathbf{7 7}$ & $\mathbf{7 7 . 0}$ & & \\
\hline Positive & 34 & 34.0 & 14 & 14.0 & & \\
\hline
\end{tabular}

Table (7): Relationship between pediatric nurses' socio demographic data and their attitudes regarding the importance of family presence in nursing care $(n=100)$.

\begin{tabular}{|c|c|c|c|c|c|c|c|}
\hline \multirow{2}{*}{ Socio demographic data } & \multicolumn{2}{|c|}{ Negative } & \multicolumn{2}{|c|}{ Neutral } & \multicolumn{2}{|c|}{ Positive } & \multirow{2}{*}{ P. value } \\
\hline & No & $\%$ & No & $\%$ & No & $\%$ & \\
\hline \multicolumn{8}{|l|}{$\overline{\text { Age }}$} \\
\hline $20<30$ & 9 & 100.0 & 63 & 81.8 & 9 & 64.3 & \multirow{3}{*}{$<0.001 * *$} \\
\hline $30<40$ & 0 & 0.0 & 9 & 11.7 & 0 & 0.0 & \\
\hline 40 years and more & 0 & 0.0 & 5 & 6.5 & 5 & 35.7 & \\
\hline \multicolumn{8}{|l|}{ Sex } \\
\hline Female & 5 & 55.6 & 56 & 72.7 & 14 & 100.0 & \multirow{2}{*}{$0.035^{*}$} \\
\hline Male & 4 & 44.4 & 21 & 27.3 & 0 & 0.0 & \\
\hline \multicolumn{8}{|l|}{ Level of education } \\
\hline Bachelor of nursing & 9 & 100.0 & 20 & 26.0 & 4 & 28.6 & \multirow{3}{*}{$<0.001 * *$} \\
\hline Secondary school diploma & 0 & 0.0 & 29 & 37.7 & 5 & 35.7 & \\
\hline Diploma of technical institute & 0 & 0.0 & 28 & 36.4 & 5 & 35.7 & \\
\hline \multicolumn{8}{|l|}{ Clinical position: } \\
\hline Head pediatric nurse & 0 & 0.0 & 22 & 28.6 & 4 & 28.6 & \multirow{3}{*}{$<0.001 * *$} \\
\hline Supervisor & 5 & 55.6 & 55 & 71.4 & 10 & 71.4 & \\
\hline Bedside pediatric nurse & 4 & 44.4 & 0 & 0.0 & 0 & 0.0 & \\
\hline Years of experience & & & & & & & \\
\hline
\end{tabular}




\begin{tabular}{|l|c|c|c|c|c|c|c|}
\hline \multirow{2}{*}{ Socio demographic data } & \multicolumn{2}{|c|}{ Negative } & \multicolumn{2}{c|}{ Neutral } & \multicolumn{2}{c|}{ Positive } & \multirow{2}{*}{ P. value } \\
\cline { 2 - 8 } & No & $\%$ & No & $\%$ & No & \% & \\
\hline$<$ 5 Years & 4 & 44.4 & 44 & 57.1 & 4 & 28.6 & \multirow{2}{*}{$<0.001 * *$} \\
\hline 5 < 10 Years & 0 & 0.0 & 19 & 24.7 & 0 & 0.0 & \\
\hline$\geq 10$ Years & 5 & 55.6 & 14 & 18.2 & 10 & 71.4 & \\
\hline Obtaining Training courses & & & & & & & \\
\hline Yes & 5 & 55.6 & 56 & 72.7 & 4 & 28.6 & \multirow{2}{*}{$<0.001 * *$} \\
\hline No & 4 & 44.4 & 21 & 27.3 & 10 & 71.4 & \\
\hline
\end{tabular}

Chi-squire test, * statistically significant difference ( $p<0.05)$, ** highly statistically significant difference ( $p<0.01)$

Table (8): Relationship between parents' and their children socio-demographic data and their attitudes regarding the importance of family presence in nursing care

\begin{tabular}{|c|c|c|c|c|c|c|c|}
\hline \multirow{2}{*}{ Socio demographic data } & \multicolumn{2}{|c|}{ Negative } & \multicolumn{2}{|c|}{ Neutral } & \multicolumn{2}{|c|}{ Positive } & \multirow{2}{*}{ P. value } \\
\hline & No & $\%$ & No & $\%$ & No & $\%$ & \\
\hline \multicolumn{8}{|l|}{ Age } \\
\hline$\leq 30$ years & 20 & 87.0 & 21 & 48.8 & 24 & 70.6 & \multirow{2}{*}{$<0.001 * *$} \\
\hline 31-45 Years & 3 & 13.0 & 22 & 51.2 & 10 & 29.4 & \\
\hline \multicolumn{8}{|l|}{ Gender } \\
\hline Fathers & 0 & 0.0 & 3 & 7.0 & 0 & 0.0 & \multirow{2}{*}{0.129} \\
\hline Mothers & 23 & 100.0 & 40 & 93.0 & 34 & 100.0 & \\
\hline \multicolumn{8}{|l|}{ Level of education } \\
\hline Secondary & 20 & 87.0 & 32 & 74.4 & 21 & 61.8 & \multirow{3}{*}{0.214} \\
\hline Technical qualification & 3 & 13.0 & 6 & 14.0 & 7 & 20.6 & \\
\hline University degree & 0 & 0.0 & 5 & 11.6 & 6 & 17.6 & \\
\hline \multicolumn{8}{|l|}{ Residence } \\
\hline Urban & 6 & 26.1 & 16 & 37.2 & 10 & 29.4 & \multirow{2}{*}{0.603} \\
\hline Rural & 17 & 73.9 & 27 & 62.8 & 24 & 70.6 & \\
\hline \multicolumn{8}{|l|}{ Child been in hospital before } \\
\hline Yes & 14 & 60.9 & 24 & 55.8 & 14 & 41.2 & \multirow{2}{*}{0.276} \\
\hline No & 9 & 39.1 & 19 & 44.2 & 20 & 58.8 & \\
\hline \multicolumn{8}{|c|}{ Previous experience of hospitalization } \\
\hline Yes & 0 & 0.0 & 19 & 44.2 & 12 & 35.3 & \multirow{2}{*}{$<0.001 * *$} \\
\hline No & 23 & 100.0 & 24 & 55.8 & 22 & 64.7 & \\
\hline \multicolumn{8}{|l|}{ Child's age } \\
\hline$<5$ years & 23 & 100.0 & 32 & 74.4 & 34 & 100.0 & \multirow{3}{*}{$<0.001 * *$} \\
\hline 5-10 Years & 0 & 0.0 & 6 & 14.0 & 0 & 0.0 & \\
\hline 10 Year or more & 0 & 0.0 & 5 & 11.6 & 0 & 0.0 & \\
\hline \multicolumn{8}{|l|}{ Gender of the child } \\
\hline Male & 18 & 78.3 & 24 & 55.8 & 25 & 73.5 & \multirow{2}{*}{0.110} \\
\hline Female & 5 & 21.7 & 19 & 44.2 & 9 & 26.5 & \\
\hline \multicolumn{8}{|l|}{ child's birth order } \\
\hline The first & 9 & 39.1 & 10 & 23.3 & 11 & 32.4 & \multirow{5}{*}{0.079} \\
\hline Second & 8 & 34.8 & 11 & 25.6 & 5 & 14.7 & \\
\hline Third & 6 & 26.1 & 13 & 30.2 & 6 & 17.6 & \\
\hline Fourth & 0 & 0.0 & 6 & 14.0 & 10 & 29.4 & \\
\hline Fifth or more & 0 & 0.0 & 3 & 7.0 & 2 & 5.9 & \\
\hline
\end{tabular}

Chi-squire test, * statistically significant difference $(p<0.05), * *$ highly statistically significant difference $(p<0.01)$

Table (1): Three fourths $(75 \%)$ of the pediatric nurses in this study were females and their ages divided into three different groups, the majority of them $(81 \%)$ were from $20<30$ years. Regarding level of education, more than one fourth of pediatric nurses were secondary school diploma. More than two thirds $(70 \%)$ were supervisor in their clinical 
position, and more than half of the pediatric nurses $(52 \%)$ their years of experience were $<5$ years

Table (2): Regarding the socio demographic data of parents, majority of parents $(97 \%)$ were mothers and only $3 \%$ were fathers, near two thirds of them $(65 \%)$ their ages were 30 years or less, near three fourth of them $(73 \%)$ were secondary level of education, more than two thirds of parents $(68 \%)$ were from the rural area, more than half of them $(52 \%)$ their children were hospitalized before, $67 \%$ of their children were males and the majority (89\%) was aged from 1-5 years.

Table (3): presented percentage distribution of pediatric nurses' attitude regarding the importance of family in pediatric nursing care. In the subscale: Family as a resource in nursing care, nearly three fourths of pediatric nurses $(73 \%)$ agree with item I gain a lot of worthwhile knowledge from families which I can use in my work, while $65 \%$ of them disagree with item getting involved with families gives me a feeling of being useful, and more than half of pediatric nurses (56\%) agree with item the presence of family members is important to me as a pediatric nurse. In the subscale: Family as a burden, $49 \%$ of pediatric nurses agree that the presence of family members makes me feel stressed, and $49 \%$ strongly agree with the item of I don't have time to take care of families.

In the subscale: Family as a conversational partner, more than two thirds of pediatric nurses $(69 \%)$ agree with item it is important to find out what family members a patient has, and more than half of them (58\%) agree with item I invite family members to have a conversation at the end of the care period. In the subscale: Family as own resource, more than three fourth of pediatric nurses $(78 \%)$ agree with item it I consider family members as co-operating partners and more than two thirds of them (67\%) agree with item I ask families how I can support them (Table 3). Table (4): Displayed the mean score of the general pediatric nurses' attitude as regards FINC-NA scale and the mean of its subscale. The mean score of the general pediatric nurses' attitude was $49.6 \pm 6.96$. The mean score for subscale family as resources in nursing care was $17.65 \pm 4.43$. In the subscale family: burden the total mean score was $15.92 \pm 3.4$, in the subscale family as a conversational partner the mean score was $7.83 \pm 2.02$ and the mean score of the subscale family as own resource was 8.2 \pm 1.51 .

Table (5): Showed percentage distribution of parents' attitudes regarding the importance of family in pediatric nursing care. Most of parents (95\%) prefer the presence next to their children in any of the nursing procedures, but three fourth of them (75\%) reported that they think that there is no need for their presence during invasive painful procedures, also nearly two thirds of parents $(63 \%)$ said they cannot see their children in pain during nursing procedures more than two thirds of parents $(71 \%)$ reported that, the pediatric nurse prepares the mothers to support their children during all nursing procedures.

Table (6): Displayed parents versus pediatric nurses' general attitude regarding the importance of family in pediatric nursing care. Highly statistical significant difference were found between pediatric nurses and parent's attitudes ( $\mathrm{p}<0.001 * *)$.

Table (7): Illustrated relationship between pediatric nurses' socio demographic data and their attitudes regarding the importance of presence the family in pediatric nursing care. Highly statistical significance differences were found between pediatric nurses' socio demographic data such as age, education level, clinical position, year of their experience in pediatric clinics and their attitude regarding the importance of presence the family in pediatric nursing care $(\mathrm{P}<0.001 * *)$.

Table (8): Revealed relationship between parents' socio demographic data and their attitudes regarding the importance of presence the family in pediatric nursing care. Highly statistical significance differences were found between parents' socio demographic data as regard to their age, age of the child and their attitude regarding the importance of families involvement in care given for their children $\left(\mathrm{P}<0.001^{* *}\right)$. But no statistical significance differences were found as regard to parents' gender, level of education, residence, and if the child hospitalized before.

\section{Discussion}

Parents are the natural caregivers for their children. During their child's critical illness, much of the care giving role of parents is transferred to health professionals, because of the complex care required. This alteration of parental role has been recognized as the highest source of stress among parents. Parents need to be recognized as important to their child's recovery and contribute in their child's care (MacDonald et al., 2012) While pediatric nurses start to take care of the child in the hospital, parents lose their sense of control. At this point, concept of family centered care appears and pediatric nurses contribute the care of child with their families (Tufekci et al., 2015) So, the current study aimed to assess pediatric nurses' versus parents' attitudes regarding the importance of families' involvement in care given for their children.

In our study, three fourths of the pediatric nurses were females, more than one fourth of them were secondary school diploma and more than half of them their experience were less than five years. These results were in agreement with other study conducted 
by Mohammed et al., 2013 who stated that, $66 \%$ of them were graduated from secondary school of nursing. The mean of the nurses' years of experience in caring for pediatric patients was 11.31 \pm 8.46 . More than half $(56 \%)$ of them were staff bedside nurses.

Regarding the socio demographic data for parents, the result of this study revealed most of parents were mothers, this may be related to the mothers are usually present with their children in hospital for caring them, while fathers are stay at home with other siblings and continuing to work, near two thirds of them their ages were 30 years or less. Nearly three fourths of them were secondary level of education. These results were agreed with the results of Pinheiro de Melo et al., (2014) who stated that, the caregiver sample was composed of mothers $(82 \%)$, fathers $(17 \%)$ with only $1 \%$ consisting of other persons. The average age was 33 years old, with complete prime school or incomplete middle school (63\%).

In the present study, more than half of parents reported that their children were hospitalized before, and they had previous inpatient experience with another child. These results were in agreement with another study conducted by Gill et al., (2014) who found that, most parents reported their hospitalized child had at least one previous admission to the hospital, and most of them reporting a previous experience in hospital with another child.

Related to the parents' attitude regarding the importance of family in pediatric nursing care, three fourth of parents reported that, they think that there is no need for their presence during invasive painful procedures, also nearly two thirds of them said they cannot see their children in pain during nursing procedures, while most of them reported that they prefer the presence beside their children in any of the nursing procedures otherwise painful procedures. In our opinion these results may related to parents when participate in any nursing procedures that cause pain to their children, this gives them a painful feeling because they feel grief when their children are exposed to this painful situation.

In the present study, highly statistical significance differences were found between pediatric nurses' socio demographic data such as age, education level, clinical position, year of their experience in pediatric clinics and their attitudes regarding the importance of family involvement in pediatric nursing care. These results were agreed with results of another study showed by Rostami et al., (2015) who stated that, there were no significant relationship among the pediatric nurses' socio-demographic characteristics and their attitudes. But these results were discrepant the results of Kurtulus et al., (2018) who found no effect of pediatric nurses' demographic factors on their parent participation attitudes.

Results obtained showed that more experienced pediatric nurses had higher scores on the FINC-NA scale, which indicate positive attitudes regarding the importance of family presence in pediatric nursing care. This result is matched with other studies, which revealed that more experienced pediatric nurses are valued family care more highly than younger and less experienced pediatric nurses (Sveinbjarnardottir et al., 2011) In my view this is may be related to more experienced pediatric nurses had more previous experience with mothers' presence which reflect more accepting of the presence of mothers in pediatric procedures.

More than three fourths of pediatric nurses in this study showed neutral attitude regarding the importance of involving families in nursing care. As regard to parent's attitudes nearly half of them were also neutral attitude. Al-Mutair, et al., (2012) reported in his study negative attitudes of health care workers including pediatric nurses toward family presence. While the same results were found in a study that used the same scale by Rostami et al., (2015) who stated that, most of pediatric nurses had neutral attitudes toward family participation in their children's care. But these findings are in contrast to other studies conducted by Angelo et al., (2014) \& Fernandes et al., (2015) who found that, most participating pediatric nurses showed a supportive attitude concerning the importance of including families in nursing care.

From the researchers' point of view, these results were not fully desired as we would like about nurses and parents' attitude to be more positive, these results may be related to lack in their awareness about the significance of including families in child nursing care. So the researchers saw that both nurses and parents need in-service training programs regarding the importance of involving families in child nursing care.

Highly statistically significant differences were found between pediatric nurses and parent's attitudes. This result was agreed with the result of Abdulbaki et al., (2011) who indicated that, a statistically significant difference among mothers and pediatric nurses' attitude towards mothers' participation in some aspects of child's moral support activities. But these results were opposed the result found in other study of Mohammed et al., (2013) who stated that, there was no statistically significant difference was detected between mothers and pediatric nurses' general attitude.

In this study the mean score of the general pediatric nurses' attitude as regards FINC-NA scale indicated that pediatric nurses had a neutral attitude regarding 
the importance of families' involvement in nursing care. This result was opposed the result found by Benzein et al., (2008) who stated that the mean score of the total FINC-NA scale indicated that pediatric nurses who worked in the place had a supportive attitude regarding the importance of families in nursing care.

In the current study, the mean score for subscale family as resources in nursing care was 17.6. In the subscale family as a conversational partner, the mean score was 7.8. In the subscale family as a burden, the total mean score was 15.9 . In the subscale family as own resource the mean score was 8.2. These results were approved with another study conducted by Benzein et al., (2008) who found that, the median score for the subscale Family as a resource in nursing care was 36 , the median score for the subscale family as a conversational partner was 27 , the median score for the subscale family as a burden was 13 , and the median score for the subscale family as own resource was 13 .

In our study, fewer of pediatric nurses and more than one third of parents' attitude were had positive attitude and there was highly statistically significant difference between the attitude of the pediatric nurse and the parents' attitude regarding the importance of families' involvement in nursing care. These results was in opposite of the study results which conducted by Mohamed et al., (2013) who showed that, the mothers had less positive attitude than pediatric nurses and there was no statistically significant difference between the mothers and pediatric nurses' attitude in relation to the presence of mothers with their hospitalized children.

\section{Conclusion}

The present study concluded that, more than three fourths of pediatric nurses and nearly half of parents showed neutral attitude regarding the importance of families involvement in care given for their children. Most of parents reported that they prefer the presence beside their children in any of the nursing procedures otherwise painful procedures. There was no statistically significant difference between the parents' and pediatric nurses' attitude in relation to the presence of them with their hospitalized children.

\section{Recommendations}

Based on the findings of the present study the following recommendations are suggested:

1- In service training programs should be provided for all categories of pediatric nurses regarding the importance of involving families in nursing care.

2- Parents of hospitalized children should be provided that with the chances to participate in their children's care.
3- The study must be replicated to conduct on a larger sample including other pediatric hospitals are needed for generality of the reached results.

\section{References}

1. Abdulbaki M., Gaafar Y., \& Waziry O., (2011): Maternal Versus Pediatric nurses Attitudes Regarding Mothers' Participation in the Care of Their Hospitalized Children. Journal of American Science, 7(9):316-327.

2. Al Mutair A., Plummer V., \& Copnell B., (2012): Family Presence During Resuscitation: Descriptive Study of Pediatric nurses' Attitudes from Two Saudi Hospitals. Nursing in Critical Care; 2012 :17(2):90-8.

3. Angelo M., Cruz A., Mekitarian F., Santos C., Martinho M., |\& Martins M., (2014): Pediatric Nurses' Attitudes Regarding the Importance of Families in Pediatric Nursing Care. Revista da Escola de Enfermagem da USP, vol.48 no.spe São Paulo Aug..

4. Atay G., Eras Z., \& Ertem, I., Developmental Support of Children during Their Hospitalizations. Cocuk Dergisi (The Journal of the Child) 11(1):1-4.

5. Ball J., Bindler R., \& Cowen K., (2013): Principles of Pediatric Nursing: Caring for Children, 5th ed., New York: Pearson Company, 2013:20, 263-289.

6. Bell J., \& Wright L., (2011): The Illness Beliefs Model: Creating practice knowledge in family system nursing for families experiencing illness. In E.K. Svavarsdottir \& H. Jonsdottir (Eds.), Family nursing in action. Reykjavik, Iceland: University of Iceland Press, 2011:1551.

7. Benzein E., Johansson P., Årestedt K., \& Britt-Inger Saveman B., (2008): Pediatric Nurses' Attitudes about the Importance of Families in Nursing Care a Survey of Swedish Pediatric nurses. Journal of Family Nursing, 14 (2): May 2008 162-180.

8. Chung L., Ho K., \& Kwok B., (2016): Play interventions to reduce anxiety and negative emotions in hospitalized children. BMC Pediatrics 2016; 16(1): 36.

9. Dur S., Gozen D., \& Bilgin M., (2016): Attitudes and Behavior of Pediatric nurses in State and Private Hospitals with Respect to Family-Centered Care. Journal of Current Pediatric 2016; 14:1-9.

10. Fernandes C., Gomes J., Martins M., Gomes B., \& Gonçalves L., (2015): The Importance of Families in Nursing Care: Pediatric nurses' Attitudes in the Hospital Environment. Journal of Nursing Referência. IV 2015; 21(7):30. 
11. Gill F., Pascoe E., Monterosso, L., Young J., Burr C., Tanner A., \& Shields L., Parent and staff perceptions of family centered care in two Australian children's hospitals. European Journal for Person Centered Healthcare, 2014; 2 (1): 317-325.

12. Holmgren J., Emami A., Eriksson L., \& Eriksson H., (2014): Intersectional perspectives on family involvement in nursing home care: rethinking relatives' position as a between ship. Nursing Inquiry, 2014; 21(3): 227-237. doi:10.1111/nin.12046.

13. Kim Y., \& Cho H., (2017): Convergence Study on Nursing Needs of Hospitalized Children's Mothers and Quality of Care in Pediatric Unit. Journal of the Korea Convergence Society 2017; 8(10):155-166.

14. Kurtulus N., Yıldız K., Korucu E., \& Ozyazicioglu N., (2018): Determination of Pediatric nurses' Attitudes on Family-Centered Care. International Journal of Caring Sciences May-August 2018; 11(2):1066.

15. MacDonald M., Lien S., \& Carnival F., (2012): An office or a bedroom? Challenges for family-centered care in the pediatric intensive care unit. Journal of Child Health Care. 2012; 16(3):237-49.

16. Mohammed A., Mohammed S., \& Abu Salem L., (2013): Mothers Versus Pediatric nurses' Attitude Regarding Mothers' Presence during Invasive Nursing Procedures for Their Hospitalized Children. Life Science Journal 2013; 10(3):379-388.

17. Oliveira P., Fernandes H., Vilar A., Figueiredo M., Ferreira M. \& Martiho M., (2011): Attitudes of pediatric nurses towards families: Validatin of the scale Families Importance in Nursing Care - Pediatric nurses Attitudes. Revista da Escola de Enfermagem da USP. 2011 [cited 2014 Mar 22]; 45(6):1329-35.

18. Pinheiro de Melo E., Ferreira O., \& Garcia de Lima R., (2014): The involvement of parents in the healthcare provided to hospitalzed children. Rev. Latino-Am. Enfermagem vol.22 no.3 Ribeirão

Preto May/June 2014.

19. Rostami F., Hassan S., Yaghmai F., Ismaeil S., \& BinSuandi T., (2015): The Effect of Educational Intervention on Pediatric nurses' Attitudes Toward the Importance of FamilyCentered Care in Pediatric Wards in Iran. Electron Physician. 2015 September; 2015; 7(5): 1261-1269.

20. Saveman B., Benzein E., Engström Å., \& Årestedt K., (2011): Refinement and psychometric reevaluation of the instrument:
Families' importance in nursing care--pediatric nurses' attitudes. Journal of Family Nursing 2011; 17(3):312-329.

21. Sveinbjarnardottir E., Svavarsdottir E., \& Saveman B., (2011): Pediatric nurses' attitudes towards the importance of families in psychiatric care following an educational and training intervention program. Journal of Psychiatric and Mental Health Nursing. 2011; 18(10):895-903.

22. Tufekci, F., Tosun, A., Aile C., Bakim M., \& Incelenmesi U., (2015): The Examination of Family Centered Care Practices in Pediatric Clinics. Journal of Anatolia Nursing and Health Sciences.2015; 18(2): 131-139. 